\title{
Active surveillance before radiotherapy — outcome and predictive factors for multiple biopsies before treatment
}

\author{
Alexandre Alcaidinho' ${ }^{1}$; Guila Delouya ${ }^{1}$; Jean-Paul Bahary ${ }^{1}$; Fred Saad ${ }^{2}$; Daniel Taussky ${ }^{1}$ \\ ${ }^{1}$ Department of Radiation Oncology, Centre hospitalier de l'Université de Montréal, Montreal, QC, Canada; \\ ${ }^{2}$ Division of Urology, Centre hospitalier de l'Université de Montréal, Montreal, QC, Canada
}

Funding: This study was supported by Sanofi Canada.

Cite as: Can Urol Assoc J 2020 July 17; Epub ahead of print. http://dx.doi.org/10.5489/cuaj.6523

Published online July 17, 2020

$* * *$

\section{Abstract}

Introduction: We aimed to investigate whether patients on active surveillance (AS) had worse outcomes than patients who received immediate treatment with radiotherapy and whether a Gleason grade progression on repeat biopsy influenced outcome.

Methods: From our institutional database, we identified 2001 patients treated between 2005 and 2019 with primary external beam radiation therapy or brachytherapy. Biochemical recurrence (BCR) was analyzed in relation to clinical factors such as a Gleason grade progression or having multiple biopsies vs. only one biopsy. Patients on AS were identified as those who had undergone $\geq 2$ biopsies. We used log-rank tests for univariate analysis (UVA) and Cox regression analysis for multivariable analysis (MVA).

Results: Of 2001 patients, 374 (19\%) patients had $\geq 2$ biopsies before treatment, of which $48 \%$ presented with a Gleason grade progression of mostly to Gleason $3+4(36 \%) ; 32 \%$ had a cancer volume increase on biopsy and $16 \%$ had no significant change on biopsy. For patients with $\geq 2$ biopsies, median time from first biopsy to treatment was 22.0 months (interquartile range [IQR] 14.7-36.1). By UVA, patients with Gleason grade progression $(n=105)$ had a worse BCR-free rate $(p=0.02)$ than patients who had no grade progression on repeat biopsy or only one biopsy. On MVA, this effect was lost. Having $\geq 2$ biopsies was not a significant negative prognostic factor on UVA $(p=0.2)$ or MVA.

Conclusions: In our experience, radiotherapy after a period of AS, even with Gleason grade progression, did not lead to worse outcomes compared to patients who had radiotherapy after only one biopsy. 


\section{Introduction}

Active surveillance (AS) is a recommended and widely used option in prostate cancer for patients with low-risk disease and for selected patients with low-intermediate risk cancers ${ }^{1}$. AS has demonstrated excellent results in a large Canadian cohort ${ }^{2}$ of patients with very low 15 -year prostate cancer mortality. However, in this study, the outcome in patients who underwent external beam radiotherapy (EBRT) after initial AS were not as favourable with $24 \%$ experiencing biochemical recurrence (BCR). Two other studies ${ }^{3},{ }^{4}$ showed equally poor results In the so far largest study on EBRT after AS, Berlin et al. found no difference in BCR

In this retrospective study we evaluated the outcome in patients who were on AS and subsequently received curative radiotherapy by studying the impact of AS in a real-world setting. Since patients on AS came from many different centers, we arbitrarily defined patients undergoing AS as having had at least 2 biopsies before treatment and compared them to patients who received treatment after their initial biopsy.

\section{Methods}

\section{Patient cohort and clinical data}

Research and ethics board approval was obtained for this study. From our institutional database, we identified 2001 patients who had primary EBRT and/or brachytherapy from April 2005 to August 2019. All patients were entered into a prospectively maintained database. Patients treated with EBRT following radical prostatectomy (RP) were excluded. . All biopsy information was verified before data analysis.

To calculate Gleason progressions on repeat biopsy, the last biopsy was compared to the previous one, even in patients who had more than two biopsies. Biopsies analysis were categorized as developing grade progression to Gleason 3+4; Gleason 4+3; Gleason $\geq 4+4$; downgrade; volume change; and no change. The no change classification included patients who had the same Gleason score as the previous biopsy and either the same or fewer number(s) of positive biopsies than the preceding one.

BCR was calculated from end of treatment to the time of BCR as defined by the Phoenix definition (nadir+2). For all BCR analyses, only patients who had a follow-up of at least 24 months were included.

\section{Statistical methods}

Non-parametric tests (Kruskal-Wallis) or Student's t-test were used to compare the means between patient groups. Chi-square test was used to compare distributions between groups. Binary logistic regression was used to calculate factors that predict for more than one biopsy. Log-rank test was used with the Kaplan-Meier method, and Cox regression analysis was used to predict for BCR. Factors that had a $p$-value $\leq 0.1$ in univariate analysis (UVA) were included in 
multivariable analysis (MVA). Statistical significance was defined as $p$-values $\leq 0.05$. Statistical analysis was performed using SPSS 25.0 for Windows (IBM SPSS, Chicago, IL).

\section{Results}

Patient characteristics of the 2001 patients identified for this study are listed in Table 1. For 374 (19\%) patients, at least 2 biopsies were performed before definitive treatment. Of these patients, $306(82 \%)$ had 2 biopsies, 55 (15\%) had 3 biopsies, 11 (3\%) had 4 biopsies, and 1 patient each had 5 and 6 biopsies. Table 2 lists the results of the last biopsy before treatment compared to the previous one in patients who had $\geq 2$ biopsies. Notably, $48 \%$ of patients had grade progression, mainly a Gleason upgrade to $3+4(36 \%)$, whereas $32 \%$ had a cancer-volume increase on biopsy but no Gleason upgrade.

The median time from biopsy to treatment in patients with only 1 biopsy was 4.6 months (interquartile range (IQR) 3.6-6.3, standard deviation (SD) 5.1 months). For patients with $\geq 2$ biopsies, median time from first biopsy to treatment was 22.0 months (IQR 14.7-36.1) and the delay between the last biopsy and treatment start was 4.5 months (IQR 3.4-6.6, SD 5.5 months).

\section{Influence of Gleason grade progression on BCR}

A follow-up of at least 24 months without a recurrence was observed for 1401 patients, including 252 patients who had $\geq 2$ biopsies. Median time of follow-up in these patients was 52 months (IQR 36-78). Seventy-eight patients had a recurrence. The 3-year and 4-year BCR-free survival for patients with more than 1 biopsy was $99 \%$ and $98 \%$, respectively. In UVA, having $\geq 2$ biopsies was not a negative prognostic factor $(\mathrm{p}=0.2)$, but having a grade progression compared to no grade progression on repeat biopsy or only 1 biopsy was a negative prognostic factor $(p=0.02)$. Because grade progression and having at least 2 biopsies are interdependent factors, we performed a separate MVA for both factors. In the analysis on grade progression, we excluded the CAPRA score. Both $\geq 2$ biopsies and having a grade progression lost their significance (Table $3)$.

\section{Grade progression in patients with intermediate-risk cancers and a Gleason score of 7} All patients who had intermediate-risk cancers and a follow-up of at least 24 months were analyzed. We compared the 71 patients who had a grade progression to Gleason 7 with patients who had intermediate-risk cancer on initial biopsy or no grade progression on repeat biopsy $(n=602)$. Only 3 patients with a grade progression to Gleason 7 had BCR, and no difference was found between both groups $(p=0.7)$ by UVA or by MVA that had been adjusted for androgen deprivation therapy (ADT) use and type of treatment $(p=0.9)$ (detailed analysis not shown).

\section{Discussion}

Despite the widespread acceptance of AS ${ }^{1}$, not all men on AS do well. Some patients develop adverse pathological features at the time of RP, which include $\geq$ Gleason $4+3$, pT3/4 or N1 disease $^{7}$. 
Even with the strict definition of AS, the present study includes 374 patients and is the largest to date to report on the outcome of radiotherapy in patients initially on AS. Of all patients with $\geq 2$ biopsies, $48 \%$ had a grade progression to either Gleason score 7 or 8 .

We found that grade progression on repeat biopsy was not necessarily a negative prognostic factor; patients who had a Gleason grade progression had worse outcomes only on UVA, but not on MVA adjusted for treatment type. Presumably, Gleason grade progression would lead to more aggressive treatments, however, this was not the case. The use of ADT was less likely ( $4 \%$ vs. $12 \%, \mathrm{p}<0.001)$ in patients with multiple biopsies and a brachytherapy boost was as frequently used in both groups (16\% vs. 17\%).

There are few studies about EBRT after AS. In a Canadian cohort from Klotz et al. ${ }^{2}$, of the 148 patients who were treated with EBRT, the BCR-free survival rates at 5, 10, and 15 years were $79 \%, 60 \%$, and $48 \%$, respectively. In the Johns Hopkins cohort of 96 patients treated with EBRT, $15 \%$ experienced BCR ${ }^{3}$ after a median follow-up of 2.8 years after treatment. In a more recent publication, the 10 -year BCR rate $(n=45)$ after EBRT was $47 \%$ compared with $25 \%$ for radical $R P(n=27){ }^{4}$. In the single center study by Berlin et al. ${ }^{5}$ of 215 patients, they found that the BCR rate was not different between patients who were treated immediately compared to patients on AS . Interestingly, in a subgroup of 66 patients who had progressed to unfavorable intermediate-risk cancer (unfavIR), the BCR rate was lower than in patients who had unfavIR on initial biopsy ( $4.5 \%$ vs $11.7 \%, p=0.017$ ). UnfavIR was defined according to the Zumsteg-Spratt definition as more than one intermediate-risk factor, primary Gleason score of 4 or more, and greater than $50 \%$ positive biopsy cores.

Within our cohort, it was difficult to identify retrospectively patients on AS and patients that were delayed treatment because of wait times from biopsy to treatment. Therefore, we notably included the $27 \%$ with a delay of $>6$ months between biopsy and treatment in the cohort of patients on AS. A longer interval between biopsy and treatment did not necessarily imply that a patient chose AS, but could indicate that the patient needed more time to decide on their treatment or was waiting to meet with several specialists before undergoing treatment. Since all AS protocols suggest a repeat biopsy at some point, we classified patients as undergoing AS if they had at least 2 biopsies before undergoing radiation treatment.

Patients want to know whether AS has the same chance of cure as immediate treatment, even if they will experience subsequently disease progression on biopsy. We have shown excellent results with radiotherapy after initial AS. A grade progression after AS may not be a reflection of increased disease aggressiveness because other studies have described initial cancers that later progressed to a more advanced stage as being less aggressive. Kovac et al. found that patients with adverse features on pathology (Gleason score $\geq 7, \geq p T 3$, or lymph node invasion) at RP but clinically less aggressive cancers before surgery had a better prognosis ${ }^{9}$. Elshafei et al. also reported that patients with low-risk prostate cancer that was detected on repeat/subsequent prostate biopsies had a lower rate of Gleason score upgrading and extraprostatic extension than patients diagnosed on initial biopsy ${ }^{10}$. After a median of more than 
4 years of follow-up, only 6 patients died from prostate cancer. Because of the low BCR rate, we assume that the influence of grade progression on metastasis-free survival or cancer-specific survival is not large and would only be detectable after many more years of follow-up. Whether patients with a Gleason $3+3$ or $3+4$ on first or repeat biopsy really needed treatment is a question beyond the scope of this paper.

Shelton et al. analyzed the outcome of 548 patients on AS in a cohort of 9 community urology practices in the US ${ }^{12}$. The median time to active treatment or lost to follow-up was 3.35 years. Interestingly, of the $57 \%$ who had a second biopsy, $44 \%$ had disease progression. This is very similar to the $48 \%$ of our patients who experienced a grade progression to Gleason 7 or 8 disease. The mean time to active treatment in their study was 21.6 months, similar to our median of 22 months between the first biopsy and treatment in AS patients.

Our data represents "real world" results, which includes some limitations. Patients in this study were sent to radiation-oncology by an urologist and therefore may represent a selection bias because there are different criteria for AS and some patients who might qualify for (further) AS are counselled by their urologist to undergo treatment. Furthermore, because there is no uniform AS protocol and criteria for AS, defining which patient was on AS from a retrospective cohort presented challenges. We also did not include the maximum percentage of core involvement, a criteria often used to offer or discourage AS ${ }^{13}$.

We were also unable to include the influence of MRI and image-targeted biopsies on progression of the number of positive biopsies. Furthermore, information such as body mass index and prostate-specific antigen (PSA) density, which have been shown to influence upgrading under AS, were not available in our database ${ }^{14}$.

Our data may not be applicable to other countries because of the universal health-care coverage of all residents in Canada, therefore eliminating cost as a decision factor in deciding for treatment. On the other hand, other reports from the US have shown that adherence to AS was not associated with insurance type ${ }^{12}$. Genomic testing is not covered by insurance and therefore was very rarely used in our patients. During the time of this analysis, MRI was not considered a standard of care and was infrequently used. Furthermore, race wasn't recorded in our database, however, we estimated that only about $5 \%$ of our patients were from visible minorities with very few African descendants.

\section{Conclusions}

BCR-free survival after radiotherapy in patients initially on AS (at least 2 biopsies) was excellent and patients who had a Gleason grade progression did not appear to have worse outcomes. Over $20 \%$ of patients who had only 1 biopsy had a delay of $>6$ months from biopsy to treatment. Whether this delay leads to worse outcome is the subject of ongoing analysis. Predicting which patient is at high risk of early progression remains a challenge when deciding to propose AS but with proper follow-up, our study suggests that it remains safe with good post-radiation therapy outcomes. 


\section{References}

1. Mahal BA, Butler S, Franco I, et al. Use of active surveillance or watchful waiting for low-risk prostate cancer and management trends across risk groups in the United States, 2010-2015. Jama. 2019;321:704-706.

2. Klotz L, Vesprini D, Sethukavalan P, et al. Long-term follow-up of a large active surveillance cohort of patients with prostate cancer. Journal of Clinical Oncology. 2014;33:272-277.

3. Tosoian JJ, Trock BJ, Landis P, et al. Active surveillance program for prostate cancer: an update of the Johns Hopkins experience. J Clin Oncol. 2011;29:2185-2190.

4. Whalen MJ, Pak JS, Lascano D, et al. Oncologic outcomes of definitive treatments for low-and intermediate-risk prostate cancer after a period of active surveillance. Clinical genitourinary cancer. 2018;16:e425-e435.

5. Berlin A, Ahmad AE, Chua ML, et al. Curative Radiation Therapy at Time of Progression Under Active Surveillance Compared With Up-front Radical Radiation Therapy for Prostate Cancer. International Journal of Radiation Oncology* Biology* Physics. 2018;100:702-709.

6. Zumsteg ZS, Spratt DE, Pei I, et al. A new risk classification system for therapeutic decision making with intermediate-risk prostate cancer patients undergoing doseescalated external-beam radiation therapy. European urology. 2013;64:895-902.

7. Balakrishnan AS, Cowan JE, Cooperberg MR, Shinohara K, Nguyen HG, Carroll PR. Evaluating the Safety of Active Surveillance: Outcomes of Deferred Radical Prostatectomy after an Initial Period of Surveillance. The Journal of urology. 2019:10.1097/JU. 0000000000000247.

8. Penney KL, Stampfer MJ, Jahn JL, et al. Gleason grade progression is uncommon. Cancer research. 2013;73:5163-5168.

9. Kovac E, Vertosick EA, Sjoberg DD, Vickers AJ, Stephenson AJ. Effects of pathological upstaging or upgrading on metastasis and cancer-specific mortality in men with clinical low-risk prostate cancer. BJU international. 2018;122:1003-1009.

10. ElShafei A, Nyame Y, Kara O, et al. More favorable pathological outcomes in men with low risk prostate cancer diagnosed on repeat versus initial transrectal ultrasound guided prostate biopsy. The Journal of urology. 2016;195:1767-1772.

11. Gondo T, Poon BY, Matsumoto K, Bernstein M, Sjoberg DD, Eastham JA. Clinical role of pathological downgrading after radical prostatectomy in patients with biopsy confirmed $\mathrm{G}$ leason score 3+ 4 prostate cancer. BJU international. 2015;115:81-86.

12. Shelton JB, Paivanas TA, Buffington P, et al. Three-year Active Surveillance Outcomes in a Contemporary Community Urology Cohort in the United States. Urology. 2019.

13. Bangma CH, Valdagni R, Carroll PR, Van Poppel H, Klotz L, Hugosson J. Active surveillance for low-risk prostate cancer: developments to date. Eur Urol. 2015;67:646648.

14. Newcomb LF, Thompson IM, Boyer HD, et al. Outcomes of active surveillance for clinically localized prostate cancer in the prospective, multi-institutional Canary PASS cohort. The Journal of urology. 2016;195:313-320. 
Figures and Tables

\begin{tabular}{|c|c|c|c|c|}
\hline & $\begin{array}{c}\text { One biopsy } \\
n=1627\end{array}$ & $\begin{array}{c}\geq 2 \text { biopsies } \\
n=374\end{array}$ & $\begin{array}{c}\text { All patients } \\
\mathbf{n}=2001\end{array}$ & $\mathbf{p}$ \\
\hline $\mathrm{Age}^{1}$, mean $(\mathrm{SD})$ & $66(6.6)$ & $66(6.2)$ & $66(6.5)$ & $0.3^{3}$ \\
\hline $\begin{array}{l}\text { Gleason score }(\%)^{2} \\
\quad 6 \\
3+4 \\
4+3 \\
8 \\
9-10\end{array}$ & $\begin{array}{c}46(751) \\
35(566) \\
11(180) \\
5(83) \\
3(47)\end{array}$ & $\begin{array}{c}46(172) \\
42(156) \\
9(35) \\
2(9) \\
0.5(2)\end{array}$ & $\begin{array}{c}46(923) \\
36(722) \\
11(215) \\
5(92) \\
2(49)\end{array}$ & $0.001^{5}$ \\
\hline $\begin{array}{l}\text { T-stage }(\%) \\
\text { T3-4 }\end{array}$ & $5(73)$ & $0.3(1)$ & $4(74)$ & $<0.001^{4}$ \\
\hline $\begin{array}{l}\text { PSA }(\mathrm{ng} / \mathrm{mL}) \text { before treatment }(\%) \\
\quad<6 \\
6-10 \\
10-20 \\
20-30 \\
>30\end{array}$ & $\begin{array}{c}45(731) \\
39(629) \\
12(200) \\
2(29) \\
2(28)\end{array}$ & $\begin{array}{c}46(171) \\
36(134) \\
16(61) \\
1(5) \\
0.5(2) \\
\end{array}$ & $\begin{array}{c}45(902) \\
38(763) \\
13(261) \\
2(34) \\
2(30) \\
\end{array}$ & $<0.001^{4}$ \\
\hline $\begin{array}{l}\geq 34 \% \text { positive biopsies in last } \\
\text { biopsy before treatment }(\%)\end{array}$ & $51(822)$ & $43(161)$ & $19(983)$ & $0.01^{4}$ \\
\hline ADT $(\%)$ & $12(193)$ & $4(15)$ & $10(208)$ & $<0.001^{4}$ \\
\hline $\begin{array}{l}\text { Median time from first biopsy to } \\
\text { treatment (months), (IQR) } \\
>12 \text { months }(\%) \\
>6 \text { months }(\%)\end{array}$ & $\begin{array}{c}4.6 \\
(3.6-6.3) \\
5(83) \\
28(453)\end{array}$ & $\begin{array}{c}22.0 \\
(14.7-36.1) \\
83(312) \\
98(368)\end{array}$ & $\begin{array}{c}5.2 \\
(3.8-9.1) \\
20(395) \\
41(821)\end{array}$ & $\begin{array}{l}<0.001^{6} \\
<0.001^{4} \\
<0.001^{4}\end{array}$ \\
\hline $\begin{array}{l}\text { Year of treatment }(\%) \\
2011 \text { or earlier } \\
2012-2015 \\
2016 \text { or later }\end{array}$ & $\begin{array}{l}32(521) \\
40(649) \\
28(457)\end{array}$ & $\begin{array}{l}22(81) \\
46(46) \\
33(33)\end{array}$ & $\begin{array}{l}30(602) \\
41(695) \\
29(490) \\
\end{array}$ & $0.001^{5}$ \\
\hline $\begin{array}{l}\text { Treatment }(\%) \\
\text { Prostate seed brachytherapy } \\
\text { Brachytherapy as a boost } \\
\text { External beam radiation therapy }\end{array}$ & $\begin{array}{l}63(1009) \\
17(275) \\
20(326)\end{array}$ & $\begin{array}{l}72(266) \\
16(58) \\
13(48)\end{array}$ & $\begin{array}{l}64(1275) \\
17(333) \\
19(374)\end{array}$ & $0.002^{5}$ \\
\hline
\end{tabular}

${ }^{1}$ At the time of treatment; ${ }^{2}$ Highest Gleason score; ${ }^{3}$ Student t-test; ${ }^{4}$ Fisher exact test; ${ }^{5}$ Chi-squared test; ${ }^{6}$ Mann-Whitney test. ADT: androgen deprivation therapy; IQR: interquartile range; PSA: prostate-specific antigen; SD: standard deviation.

Table 2. Pathology results of the last biopsy before treatment compared to the previous one in patients with $\geq 2$ biopsies 


\begin{tabular}{|l|c|}
\hline Pathology result of last biopsy & $\begin{array}{c}\geq 2 \text { biopsies } \\
\mathbf{n = 3 7 4}\end{array}$ \\
\hline Grade progression to Gleason $3+4$ & $135(36 \%)$ \\
\hline Grade progression to Gleason $4+3$ & $32(9 \%)$ \\
\hline Grade progression to Gleason $\geq 4+4$ & $11(3 \%)$ \\
\hline Downgrade & $16(4 \%)$ \\
\hline Cancer volume change & $120(32 \%)$ \\
\hline No change on pathology, other reasons ${ }^{*}$ & $60(16 \%)$ \\
\hline
\end{tabular}

"Including prostate-specific antigen increase, patient decision.

\begin{tabular}{|c|c|c|c|c|c|c|c|}
\hline & Univariate $^{\$}$ & & Multivari & e & & Multivaris & \\
\hline Factor & $\mathbf{p}^{*}$ & HR & $95 \% \mathrm{CI}$ & $\mathbf{p}^{*}$ & HR & $95 \% \mathrm{CI}$ & $\mathbf{p}^{*}$ \\
\hline \multicolumn{8}{|l|}{$\begin{array}{l}\text { Age (continuous } \\
\text { variable) }\end{array}$} \\
\hline Type of treatment ${ }^{1}$ & $<0.001$ & 1.7 & $1.3-2.3$ & $<0.001$ & 3.6 & $1.5-8.6$ & 0.005 \\
\hline $\mathrm{ADT}$ & $<0.001$ & 0.9 & $0.5-1.8$ & 0.8 & 0.71 & $0.08-6.1$ & 0.8 \\
\hline CAPRA $^{2}$ & $<0.001$ & 1.7 & $1.2-2.5$ & 0.005 & & & \\
\hline Grade progression & 0.02 & & & & 4.4 & $0.5-37.5$ & 0.2 \\
\hline$\geq 2$ biopsies & 0.2 & 0.8 & $0.4-1.6$ & 0.5 & & & \\
\hline
\end{tabular}

${ }^{1}$ Prostate seed brachytherapy; brachytherapy as a boost; external beam radiation therapy. ${ }^{2} \mathrm{Cancer}$ of the Prostate Risk Assessment score as a categorical variable (low-risk 0-2; intermediate-risk 3-5; high-risk 6-10). ${ }^{\$}$ Log-rank test. ${ }^{*}$ Values in bold when $\mathrm{p}<0.05$. ADT: androgen deprivation therapy; CI: confidence interval; HR: hazard ratio. 